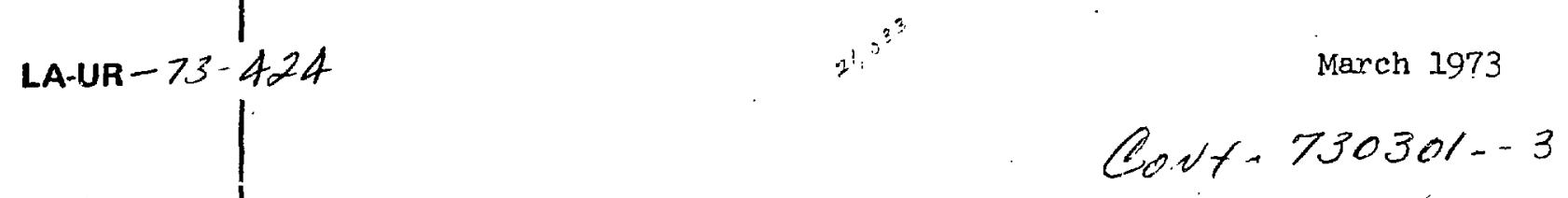

TITLE: PHOTODISINTEGRATION OF THE TRINUCLEONS

AUTHOR(S): B. F. Gibson

SUBMITTED TO: INICERHATIONAL CONFERENCE ON PHOTONUCLIPAR REACTIONS AND APPLICATIONS

By acceptance of this article for publication, the publisher recognizes the Government's (license) rights in any copyright and the Government and its authorized representatives have unrestricted right to reproduce in whole or in part said article under any copyright secured by the publisher.

The Los Alamos Scientific Laboratory requests that the publisher identify this article as work performed under the auspices of the U.S. Atomic Energy Commission.

Form No. 836

St. No. 2629

$1 / 73$
This report was preporICE

sponsored by the Urepared as an account of work the United States nor the Utates Government. Neither Commission, nor any of their employees, nor anergy their contractors, subcontractors, or their asor any of makes any warranty, express or implied, or assumes any pleteness or or resporsibility for the accuracy, com. pletiness or usefulness of any information, apparatus, product or procesa disclosed, or represents that its use

of the University of Colifornia

los alamos, NeW mexico 87544<smiles>CCCCCC</smiles>

UNITED STATES

ATOMIC ENEHGY COMMISSION CONTRACT W-7LOS-ENG. 36
Phini? :

(i) 


\title{
PHOTODISINTEGRATION OF THE TRINUCLEONS*
}

\author{
B. F. Gibson \\ Los Alamos Scientific Laboratory, University of California \\ Los Alamos, New Mexico 87544
}

\section{ABSTRACT}

A brief review of the experimental and theoretical status of the problem of the electromagnetic interactions with the trinucleon system is presented. Flastic and inelastic electron scattering as well as two-body and three-body photodisintegration are covered in some detail. Binding energy calculations and sum rule results $\left(\sigma_{-1}\right)$ are discussed and a brief introduction to the related weak interaction problem is included.

\section{INTRODUCTION}

The basic purpose of trinucleon investigations has been to add to our understanding of nucieon-nucleon (N-N) interactions. The three-nucleon system was deened especially appropriate for such studies because it was thought that, for these simplest of complex nuclei, theoretical uncertainties could be minimized. The ground state properties of $3 \mathrm{He}$ and $3_{\mathrm{H}}$ provided the first, and still important, test of those aspects of the $N-N$ interaction that cannot be examined directly in $\mathrm{N}-\mathrm{N}$ scattering experiments. The n-d scattering and breakup reactions allowed extension of the study to the twobody and three-hody continuum states. Because the photon and electron are amenable to Born approximation treatment. their interaction with the nucleus has permitted further extension of the study to include simple bound state to continuum transition processess. Here it was hoped to find even more stringent tests of the form of the $N-N$ interaction; for example, it is here that meson exchange effects appear to be suriacing.

Our purpose today is to review, very briefly, the recent experimental situation and its theoreticti interpretation. 1 The fact that the general features of the low energy three-nucleon data have been found to be somewhat insensitive to the details of the $\mathrm{N}-\mathrm{N}$ force may be a disappointment to some. On the other hand, it does indicate what success can be achieved with a correct theory and a reasonable approximation to the main features of the $N-I N$ interaction.

\section{BOUND STATE PROPERTIES}

The low energy photodisintegration and low momentum transfer electron scattering experiments are primarily sensitive to the asymptotic properties of the bound state, hence the binding energy. I wish to point out that recent variational results with local potentials, ${ }^{2}$ including all angular momentum states of the potential, indicate a bound of $7.75 \mathrm{MeV}$ for the three-nucleon binding energy for the RSC and $\mathrm{GB}$ potentials. (A check of this result by the local potential. Faddeev groups is eagerly awaited.) The axperimental triton binding energy is $8.48 \mathrm{MeV}$. However, the singlet $\mathrm{N}-\mathrm{N}$ potentials were ritted to $p-p$ scattering data, whereas $3_{H}$ has only $n-n$ and $n-p$ singlet in. teractions. Separable potential calculations indicate that the calculated binding energy of $7.75 \mathrm{MeV}$ may not be as for off as it seems: for potentials fitted to low energy scattering parameters, $v_{n n} \neq$ $V_{n p}$ adds about $0.35 \mathrm{MeV}$ to the binding energy. 3 In addition, finite size Coulomb energy calculations fail to account for the $3_{\mathrm{H}}-3_{\mathrm{He}}$ binding energy difference by 0.1 to $0.15 \mathrm{MeV}$, which may indicate a charge asymmetry in the nucleon-nucleon force. Thus, it seems possible that realistic potential calculations, for what is actually a hybrid $3_{\text {He }}$ without Coulomb interactions $\left(v^{s}=v_{p p}\right)$, should be expected to yield only about $8.0 \mathrm{MeV}$ binding.

I would also like to emphasize that the $\mathrm{N}-\mathrm{N}$ interaction must become absorptive (therefore complex) above the threshold for $\pi, 2 \pi, K$, etc. production, where $\mathrm{N}-\mathrm{N}$ scattering is no longer elastic. We mock this up in our real potential models by introducing a strong repulsion at short range. But this is only an approximation, and perhaps it is a poor one. Any calculation that depends strongly on the short range behavior of these potentials must therefore be suspect.

\section{ELECTRON SCATTERTNG}

Closely related to the binding energy 
question is the form ractor froil elastic electron scattering. This reaction, which Ieaves the ground states of $3 \mathrm{He}$ and $3 \mathrm{H}$ intact, provides a reasonable test of the charge densities resulting from the wave functions generated by the various variationa? 2 and Faddeev calculations. Because the trinucleons are spin $1 / 2$ systems, the elastic scattering data can be analysed in terms of charge and magnetic moment form factors, as is the case for the nucleons. 5 In the non-relativistic Born approximation treatment of the e-N interaction, these form factors have a simple interpretation in terms of the Fourier transforms of the charge and magnetic moment densities of the nucleus. However, meson exchange currents complicate the interpretation of these densities in terms of the trinucleon wave functions.

The most recent data 6 is for $3 \mathrm{He}$, which is shown in Fig. $I$ and 2. This experiment extended the previous measurement7 of the charge form factor beyond the region in momentum transfer $\left(q^{2}<8 \mathrm{fm}^{2}\right)$, where all reasonable three-body models fit the data, to reveal a diffraction minimum and a broad secondary maximum. In addition, the rms magnetic radius was found to be $1.95 \pm .11 \mathrm{fm}$ as opposed to the old value of $1.7^{4} \pm .10 \mathrm{fm}$.

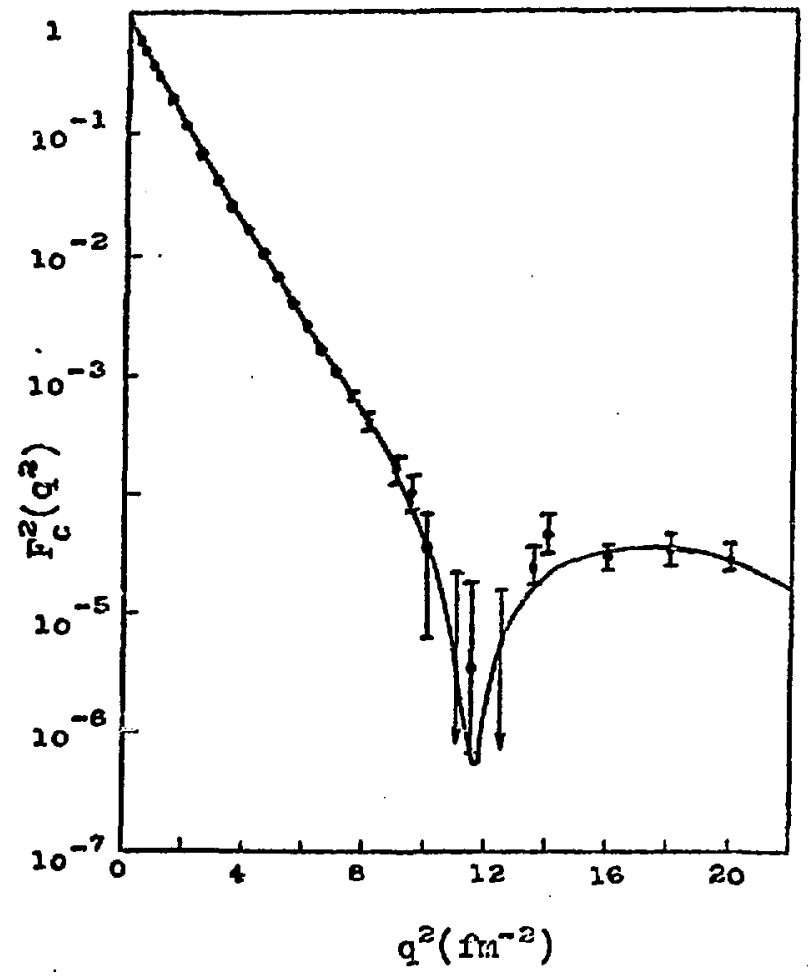

Fig. 1. The $3_{\text {He }}$ charge form factor; the curve is a. phenomenological fit yielding an rms radius of $1.88 \mathrm{fm}$.

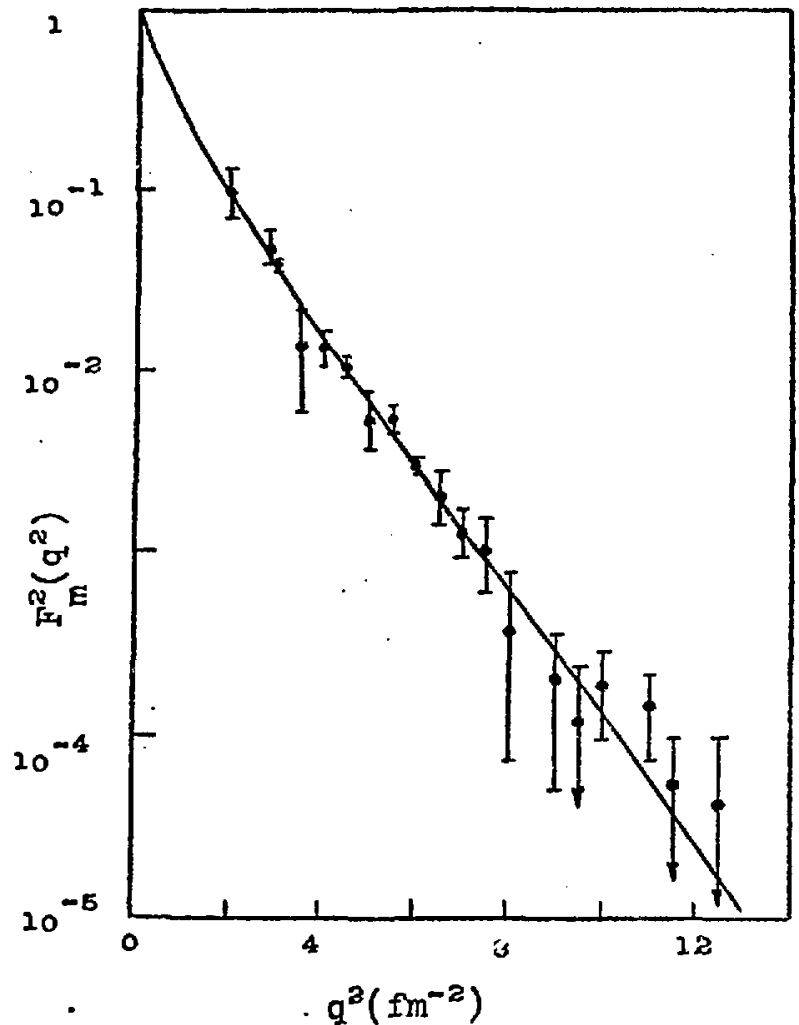

Fig. 2. The 3he magnetic moment form fretor; the curve is a phenomenological fit yielding an rms radius of $1.95 \mathrm{fm}$.

Theoretical fits to the high $q^{2}$ charge data have not been especially successful. Variational and Faddeev results (non-relativistic calculations) for the RSC potential come closer to fitting the position of the diffraction minimum than the magnitude of the second maximum. Variational results ${ }^{2}$ for the HJ and $G B$ potentials have similar short comings, as is shown in Fig. 3. Various possibilities come to mind: relativistic corrections, three-body potentials ${ }^{2}, 8$, baryon resonence contributions, etc. However, it should be noted that the two-photon exchange process is not included in this list; work on electron scattering from the deuteron 9 makes this appear to be a highly unlikely solution to the problem. Because a fit to the magnitude of the second maximum appears to be extremely difficult to obtain, this part of the data probably provides a rich source of information about the trinucleon system; however, such large momentum transfers imply a probe of very smal. internucleon distances, which is just where our $\mathrm{N}-\mathrm{N}$ interaction models are poorly approximated.

The low $q$ data appear to be more significant for classical photoruclear physics. In this reficion of momentum 


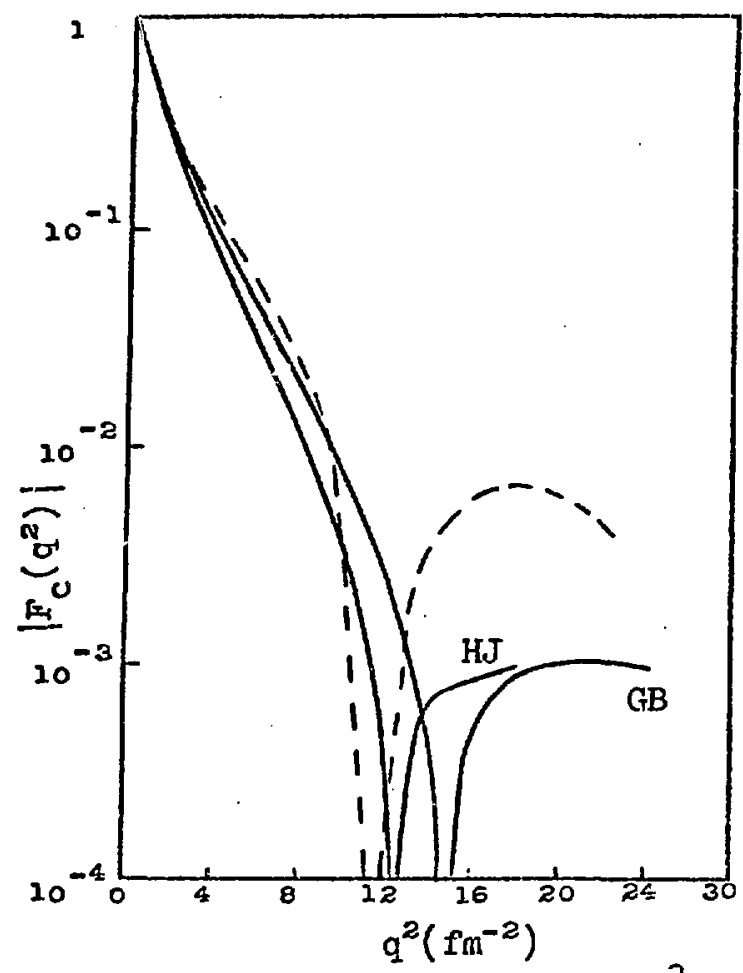

Fig. 3. Variational results for the $3_{\mathrm{He}}$ charge form factor for the HamadaJohnston and Gamme1-Brueckner potentials compared with experiment (dashed curve).

transfer one determines the shape of the charge and magnetic moment distributions that are important in the calculation of transition matrix elements. The best available data on the trinucleons determined from elastic electron scattering are listed in Table $I$.

Let us examine the radii using the form factor analysis of Schiff. 5 For the charge form factor one has:

$$
\begin{aligned}
& F_{c}\left({ }^{3} H e\right)=\left(F_{p}+\frac{I}{2} F_{n}\right) F_{1}-\frac{3}{3}\left(F_{p}-F_{n}\right) F_{2} \\
& F_{c}\left({ }^{3} H\right)=\left(F_{p}+2 F_{n}\right) F_{I}+\frac{2}{3}\left(F_{p}-F_{n}\right) F_{2},
\end{aligned}
$$

where $F_{p}$ and $F_{n}$ are the proton and neutron charge form factors and $F_{1}$ and $\mathrm{F}_{2}$ are the form factors of the $\mathrm{S}$ state and interference between the $S$ and $S^{\prime}$ states respectively:

$$
\begin{aligned}
& F_{1}=\int \exp \left(i \vec{q} \cdot \frac{2}{3} \vec{p}\right) u^{2}(r, p) d^{3} r d^{3} p \\
& F_{2}=-3 \int \exp \left(i \vec{q} \cdot \frac{2}{3} \vec{p}\right) u(r, \rho) v_{1}(r, p) d^{3} r d^{3} \rho
\end{aligned}
$$

(Defined in this way, $F_{p}>0$ for small $q$, so that $\left.\mathrm{F}_{\mathrm{C}}(3 \mathrm{He}) / \mathrm{F}_{\mathrm{c}}(3 \mathrm{HI})<\mathrm{I}_{\text {. }}\right)$ For the magnetic moment form factors one has
Table $I$. The rms radii of the trinucleons as determined by the low $q$ data of elastic electron scattering of Ref. 6 and 7.

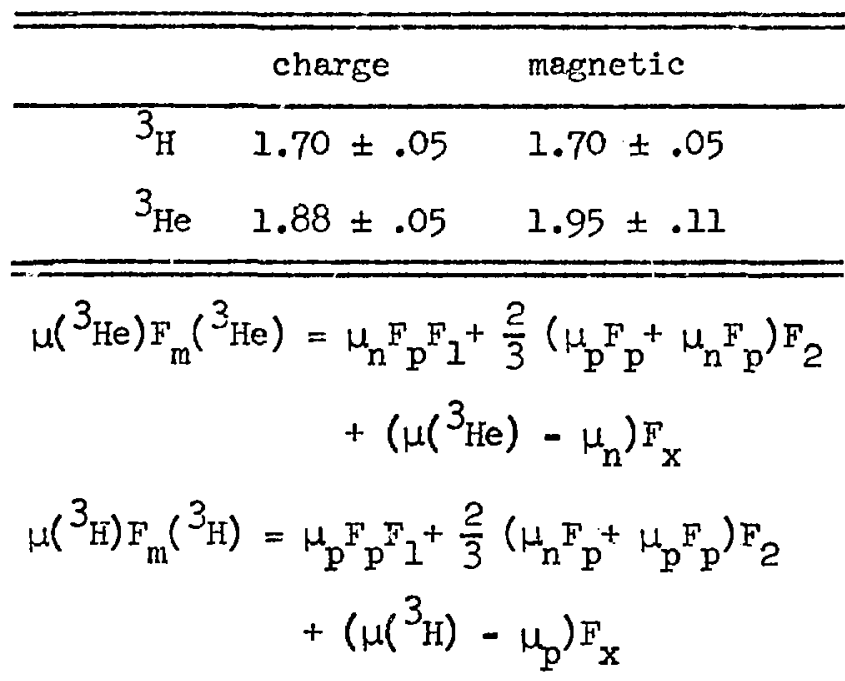

where $F_{X}$ describes the isovector exchange moment form factor and we assume

$$
F_{p}^{\text {mag }}=F_{n}^{\text {mag }}=F_{p}^{c h}=F_{p} \text {. }
$$

If, in addition, we assume for simplicity that $F_{n}=0$ and $\mu_{p}=-3 / 2 \mu_{n}$, then we can easily show that

$$
\begin{aligned}
& F_{c}\left(3_{H e}\right) / F_{p}=F_{1}-\frac{1}{3} F_{2} \\
& F_{c}\left(3_{H}\right) / F_{p}=F_{1}+\frac{2}{3} F_{2} \\
& F_{m}\left(3_{H e}\right) / F_{p}=.89\left(F_{1}-\frac{1}{3} F_{2}\right)+.11 F_{x} / F_{p} \\
& F_{m}\left(3_{H}\right) / F_{p}=.94\left(F_{1}+\frac{2}{9} F_{2}\right)+.06 F_{x} / F_{p} .
\end{aligned}
$$

From this oversimplified form factor analysis we can draw several conclusions:

1) $F_{2}>0$ implies that $r_{c}\left(3_{\mathrm{He}}\right)>r_{c}\left(3_{\mathrm{II}}\right)$;

2) $r_{m}\left(3_{\mathrm{He}}\right)>r_{c}\left(3_{\mathrm{He}}\right)$ implies that $F_{X}$ must fall. off faster than $\mathrm{F}_{\mathrm{c}}\left(3_{\mathrm{He}}\right)$, such that $r_{\mathrm{x}}>\mathrm{r}_{\mathrm{c}}\left(3_{\mathrm{He}}\right)$;

3) $r_{x}$ increases $r_{m}(3 \mathrm{He})$ relative to $r_{c}(3 \mathrm{He})$ and will have the same effect on $3 \mathrm{H}$, so that $r_{\mathrm{m}}(3 \mathrm{H})>$ $r_{\mathrm{c}}\left(3_{\mathrm{H}}\right)$;

4) from the relation

$$
\begin{aligned}
\mathrm{F}_{\mathrm{m}}\left(3_{\mathrm{H}}\right) / \mathrm{F}_{\mathrm{p}}= & .94 \mathrm{~F}_{\mathrm{c}}\left(3_{\mathrm{He}}\right) / \mathrm{F}_{\mathrm{p}} \\
& +.94\left(\frac{5}{9}\right) \mathrm{F}_{2}+.06 \mathrm{~F}_{\mathrm{x}} / \mathrm{F}_{\mathrm{p}}
\end{aligned}
$$

it is clear that $F_{2}$ and $F_{x}$ have the opposite effect, but one expects $F_{2}$ to dominate making $r_{m}\left(3_{H}\right)<r_{c}(3 H e)$. 
Thus one would expect from the data on

$3_{\mathrm{He}}$ and the above analysis that

$$
r_{m}\left({ }^{3} \mathrm{He}\right)>r_{c}\left({ }^{3} \mathrm{He}\right)>r_{m}\left({ }^{3}\right)>r_{c}\left(3_{H}\right) \text {. }
$$

Indeed, this relationship is found to hold in the Faddeev calculations--see, for example, Tjon, et al. 4 Therefore, we see that (1) the low q data are reasonably explained by a good three-body theory using $N-N$ interactions that account for the main features of the $N-N$ scattering data and (2) a remeasurement of $F_{m}(3 \mathrm{H})$ world probably shov an increases value for $r_{m}(3 \mathrm{H})$.

Inelastic electron scattering awaits the coning of precision coincidence measurements of the new accelerators. The Low momentum transfer experiments (high incident electron energy) will provide furticer information about the $3_{\mathrm{Fle}}$-pd vertex, which must be matched by valid three-nucleon bound state calculations. 10 The higher momentum transfer experiments vill begin to tell us about something other thein the asymptotic form of the trinucleon bound state--they will begin to map out the actual momentum distribution rithin the nucleus. Low energy inelastic electron scattering datali have received interpretation in terms of virtual photon theory 12 and are discussed under the section on two-body photodisintegration.

\section{PHOTODISTNTEGRATION SUM RUIES}

We have already heard from Jim O'Connell about the various sum mules that exist in photonuclear physics and related fields. Let me summarize some numerical results in the trinucleon system for the bremsstrahlung veighted sum rule. Theoretically one has 13

$$
\begin{aligned}
& \sigma_{-1}=\frac{2}{3} \pi^{2} \alpha\left(3 r_{m}^{2 l_{1}}-r_{v}^{2}\right) \\
& \sigma_{-1}\left(\frac{1}{2}\right)-\frac{1}{2} \sigma_{-1}\left(\frac{3}{2}\right)=\frac{1}{3} \pi^{2} \alpha r_{v}^{2},
\end{aligned}
$$

where the isovector radjus $r_{\mathrm{V}}$ and the matter radius $r_{m}$ are defined by

$$
\begin{aligned}
& r_{v}^{2}=\left(N r_{n}^{2}-Z r_{p}^{2}\right) / 2 \\
& r_{m}^{2}=\left(N r_{n}^{2}+Z r_{p}^{2}\right) / 3 .
\end{aligned}
$$

Calculating the isovector and matter radii from electron scattering data (the proton and neutron rms squared rodjij are assumed to be $0.0+\mathrm{fm}^{2}$ and $-0.126 \mathrm{fm}^{2}$ respective- ly) one obtains

$$
\begin{aligned}
& \sigma_{-1.1}\left(\frac{1}{2}\right)=1.34 \mathrm{mb} \\
& \sigma_{-1}\left(\frac{3}{2}\right)=1.06 \mathrm{mi} .
\end{aligned}
$$

These numbers provide an interesting comparison will the experimental data. The cloud chamber data of Gorbunov et al ${ }^{l^{4}}$ give the following:

$$
\begin{aligned}
& \sigma_{-1}(2)=1.34 \pm .05 \mathrm{mb} \\
& \sigma_{m 1}(3)=1.42 \pm .07 \mathrm{mb} .
\end{aligned}
$$

Since ve know that

$$
\begin{aligned}
\sigma_{-1 .} & =\sigma_{-1}(2)+\sigma_{-1}(3) \\
& =\sigma_{-1}\left(\frac{1}{2}\right)+\sigma_{-1}\left(\frac{3}{2}\right),
\end{aligned}
$$

it is clear that this data exceed the limit set by electron scattering. The recent data of Bernan et 2$]^{15}$ on the three-body breakup extrapolate to $\sigma_{-1}(3) \approx 1.0 \mathrm{mb}$, winich is less than the limit for $c_{\text {.... }}\left(\frac{3}{2}\right)$ '. Becanse $\sigma_{-1}(3)$ must contain all of $\sigma_{-1}\left(\frac{3}{2}\right)$, one would like a somewhat larger experimental value for $\sigma_{-1}(3)$. On the other hand, one would lilse a smaljer value for $\sigma_{-1}(2)$ than $1.34 \mathrm{mb}$, since it is unlikely that $\sigma_{-1}(2)$ contains all of $\sigma_{-1}\left(\frac{1}{2}\right)$. Such would seem to be the case if the smaller normalization for the peak two-body cross section to be discussed later is round to hold.

Theoretically, Barbour and Phillips16 have predicted in a Faddeev calculation with separable potentials trat some $15 \%$ to $20 \%$ of $\sigma_{-1}(3)$ should come from $\sigma_{-1}\left(\frac{1}{2}\right)$. On the basis of the data, this would appear to be an overestimate by a factor of 2 or so.

ANGULAR DISTRIBUTIONS. FOR $3_{\mathrm{He}}+\gamma \rightarrow \mathrm{p}+\mathrm{d}$

Some of the most precise angular distribution data in the field of photonuclear physics has been published for the $\mathrm{p}(\mathrm{d}, \gamma){ }^{3} \mathrm{He}$ reaction. 17 The statistical accuracy and the angular range of the data provide a critical test of the multipole fits predicted by any tineory. What I wish to point out here is that for data of this quality one cannot simply cut off the multipole expansion at

$$
b^{2}\left(1+\beta \cos \theta+\gamma \cos ^{2} \theta\right)
$$

and expect to obtain precision estimates 
of $\gamma$ that are meaningful theoretically. One must follow the methods of analysis required for strong interactions und include the higher partial waves, at least in Born approximation.

In Fig. 4 is show the data of Ref'. 17 for $\mathrm{E}_{\gamma}=12.1 \mathrm{MeV}$ along with a fit produced in a zero range calculation: ${ }^{18}$ the

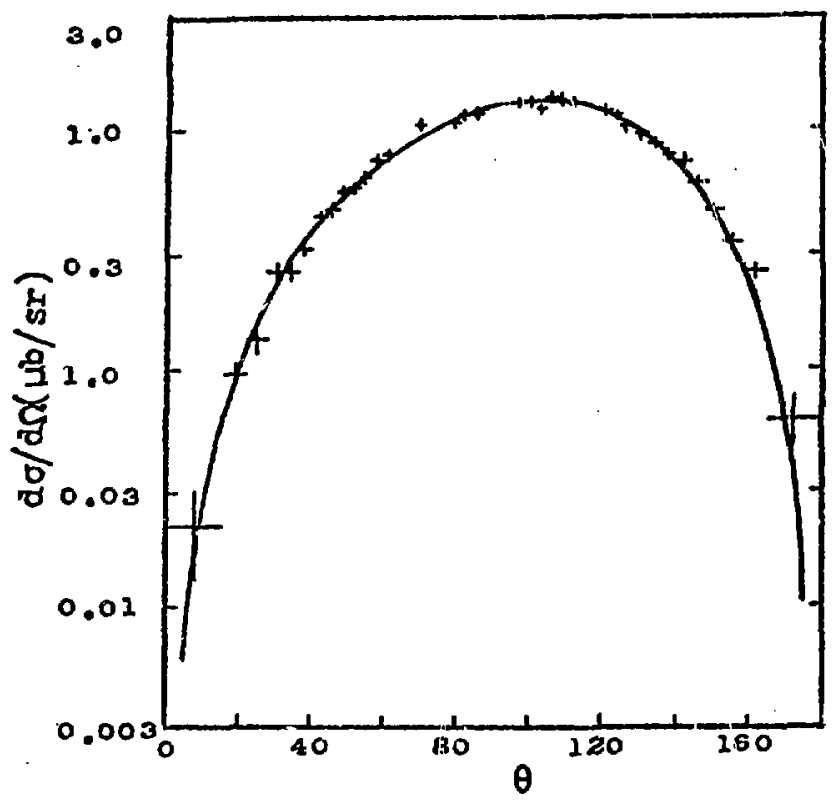

Fig. 4. Angular distribution data for the $p+d$ brealiup of 3He at an energy $E_{y}=12.1 \mathrm{MeV}$; the curve is a sum of all transitions from the $\mathrm{S}$ state of $3_{\mathrm{He}}$ to a plane wave final state of $p+d$.

curve corresponds to electric transitions from the $S$ component of the bound state to all partial waves of a plane vave final state. The $\gamma$ extracted from the data was $0.16 \pm .05$. The theoretical $y$ (obtained by expanding exact expressions for the angular distribution) is 0.09 . Despite the difference in the velues for $\gamma$, the theoretical fit j.s as good as the empirical one because the entire multipole series vas sumed. The reason for this evidently surprising difference in $y$ is clear; the expansion has not converged in three terms. Major contributions to the radial integrals in the higher partial weves $(l>1)$ come from the asymptotic region of the vave functions. (This region lies beyond the range of nuclear forces, so that a plane vave approximation to the rinal state is adequate.) The trobody separation encrgy is not large, such that the bound state wave function extends well beyond the rms radius and gives finite contributions to the integrals. Just as $(1+x)^{-1}$ is $n t$ well approximated by $1-x+x^{2}$ if one is determining the coefficient of $x^{2}$ (say for $x=0.2$ ), one cannot determine $\gamma$ properly by truncating the multipole expansion. 19 The point to be emphasized here is that, if the experimental $\gamma$ is to be interpreted properly theoretically, then it must be extracted in an analysis that includes the

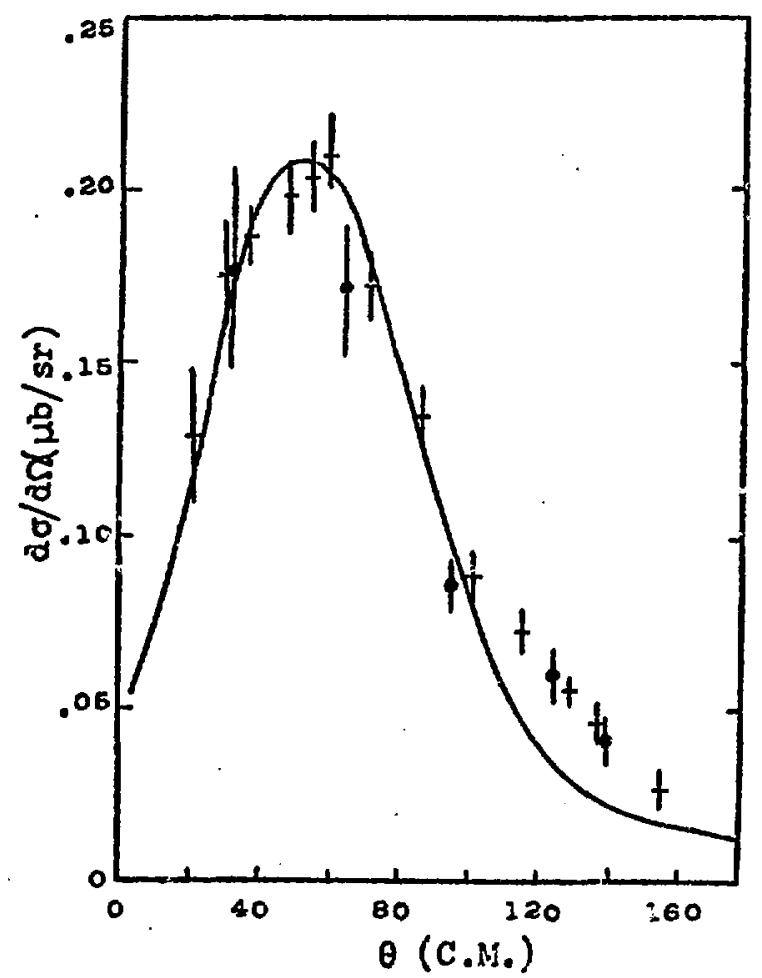

Fig. 5. Angular distribution data for the $p+d$ capture reaction corresponding to $\mathrm{E}_{\gamma}=109 \mathrm{MeV}$. The solid dets are from $0^{\prime}$ Falion et $a l$ and tile other data are from Didelez et al, Ref, 21 .

effects of the neglected terms of the multipole sum, at least in a plane wave approximation.

The data of Ref. 17 are clearly not the only angulor distribution data available. Other such data in the low energy region can be found in the papers of Ref. 14 and 20 . There would appear to be littile evidence for abnormal structure in $\beta$ 
for $E_{\gamma}<40 \mathrm{MeV}$. It seems to be a smooth function of energy as theory would predict.

At higher energies $\left(E_{\gamma}>40 \mathrm{MeV}\right)$ the angular distribution appears to exhibit a broad structure at the back angles. 21 Data corresponding to $\mathrm{E}_{\gamma}=109 \mathrm{MeV}$ are show in Pig. 5 along with a piane wave multipole fit generated by Gibson and 0 'Connell, 22 in which spin-flip and Dstate transitions were included. The broad structure at back angles has not been reproduced. This feature of the data is most pronounced in the work of o'fallon et al near an energy of $80 \mathrm{MeV}$ and appears to heve venished ky $140 \mathrm{MeV}$.

\section{do/dE' FOR THE TTOO-BODY BREAKUP}

The tro-body photodisintegration energy spectrum has proved to be somewhat of an enigma. Basically an electric dipole transition from a spatially symmetric $S$ component of the ground state to a nucleondeuteron p-wave final state, this reaction has provided much excitement over the past few years as various controversies have waxed and waned. First, there was the disagreement between the $90^{\circ}$ differential cross section measurements 23 and the total cross section measurenent ${ }^{24}$ as to the correct normalization of the peak cross section. Early theoretical estimates, 25 which treated the final state in a plane wave approximation with no deuteron distortion, tended to agree with the smaller, $90^{\circ}$ data nomalization. Faddeev theory was then utilized to estimate the cross section in terms of a simple separablepotential model; a $25 \%$ increase in the theoretical cross section was obtained. 26 Thus, the normalizetion of the total cross section measurement became favored, and new experiments tencied to arree with the higher value. 27 At about the same time apparent structure developed in the cross section below $\mathbb{E}_{\gamma}=30 \mathrm{MeV}$. This structure was difficult to understand theoretically, and the problem disappeared when the structure vanished upon remeasurement. But just as the structure question was being resolved, the normalization question arose again: a new p-d capture experiment was reported, 28 which agrees with the mggnitude of the old $90^{\circ}$ data, and a contribution to this conference reports $A$ cross section measurement that also supports this normalization. 29 In addition, Hendry and Phillips 30 have asserted that they believe the separable model may yield too large a cross section. However, the

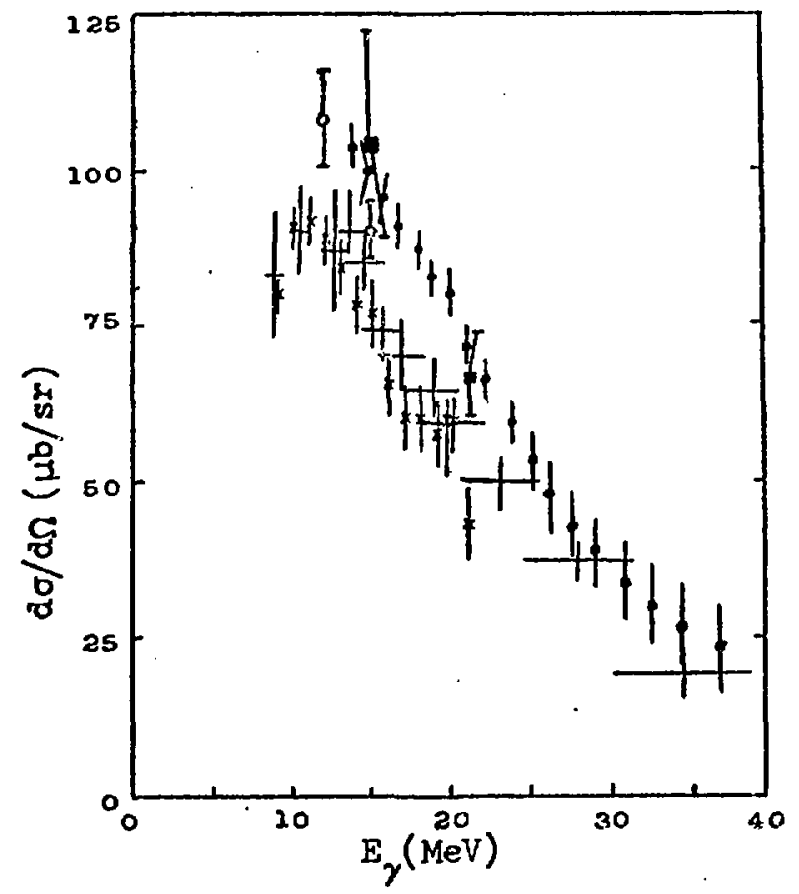

Fig. 6. Comparison of $90^{\circ}$ differential cross section measurements: $x$ Bermen, et al; + stewar', et al; o Belt, et al; Halbert, et al; - Shin, et al.

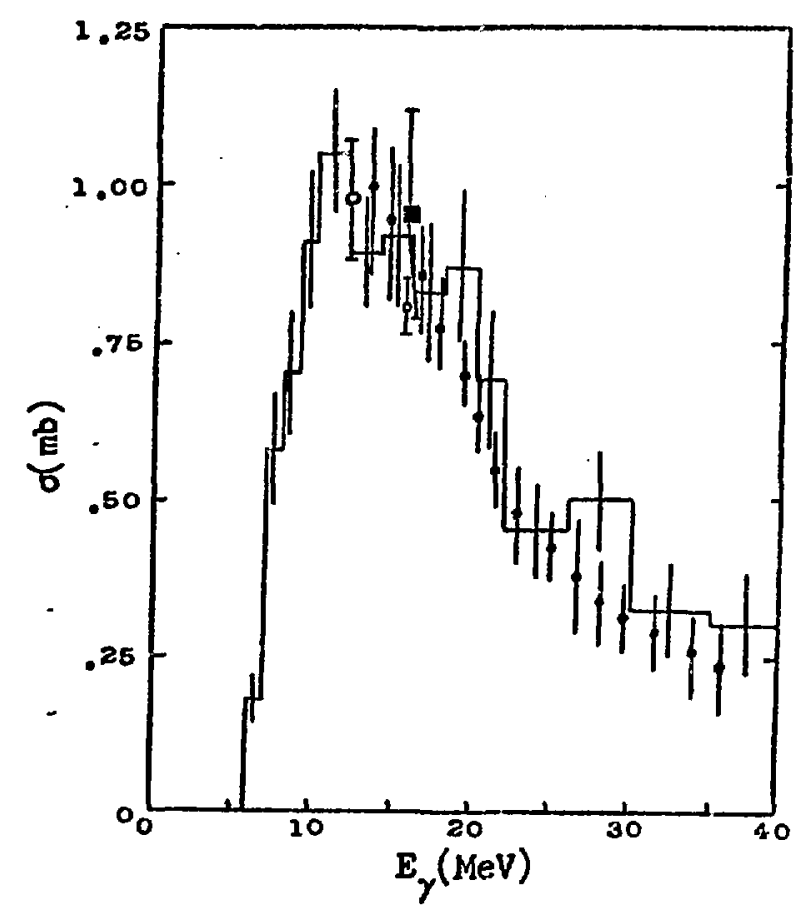

Fig. 7. Comparison of total cross section measurements: $\Omega$ Fetisov, et a].; - Belt, et al; \& iajuert, et al; - Shin, et al.

latest results from the Nationel. Bureau of Standards 31 would seem to support the higher peak cross section value and are in 
excellent agrecment with a separablepotential calculation. 3 ?

Let us examire the low energy data. Shown in Fig. 6 is a reasonable semple of the $90^{\circ}$ data available through the middle of last year. Clearly the magnitude of the peak cross section clusters about two different values. One wonders how a number of different experiments (capture, photon, and electron) can factor so neatly into two different groups. It should be noted from this figure tinat all of the experiments tend to agree above $25 \mathrm{MeV}$. In Fig. 7 we see a similer collection of date. for the total cross section measurement. The agreement as a function of time with the old total cross section data (shown as a histogram) is apparent. The value of $64 \mathrm{mb}$ reported from the recent capture measurement 28 would fall slightly below the data shom in the figure $\left(E_{\gamma}=16 \mathrm{MeV}\right)$

A sample of the unpublished data from Glasgow $33^{\circ}$ for the photodisintegration reaction is show in rig. 8 . The curve refers to a separable-potential model calculation 32 that agrees reasonably well with the data in Fig. 7. It is shown here for orientation purposes. Note the following: 1) agreement rith the data above $20 \mathrm{MeV}$ is quite good--recall that all the $90^{\circ}$ experiments shown in Fig. 6 appeared to be consistent beyond $25 \mathrm{NeV}$; 2) the calculation badly overestimates the data in the region of the peal cross section. In contrast tr this, we can see in Figg 9 the data from the Bureau of Standards 31 compared to the same theoretical calculation. Here agreement with the data from the peak cross section energy to above 20 $\mathrm{MeV}$ is clearly evident.

Although a theorist should always be wory of making remarks about experimental data, it would appear that there is some difficulty in counting the deuterons from the two-body breakup reaction below $20 \mathrm{MeV}$. All of the experiments are in reasonable agreement as to the magnitude of the cross section above $E_{\gamma}=20 \mathrm{heV}$. Since it becomes more difficult to get the deuteron out of the target as the deuteron energy becomes smaller, it would seem that the experimental problems becone greatest in the 10, energy region (around the peak cross fection). Conversations with Bill Dodge 3 ! indicate thet, at least in the experiments done at the llational Bureau of Standards, very thin-walled torgets must be employed if an o.ccurate ineasurement of the loit enerey deuterons is to be made.

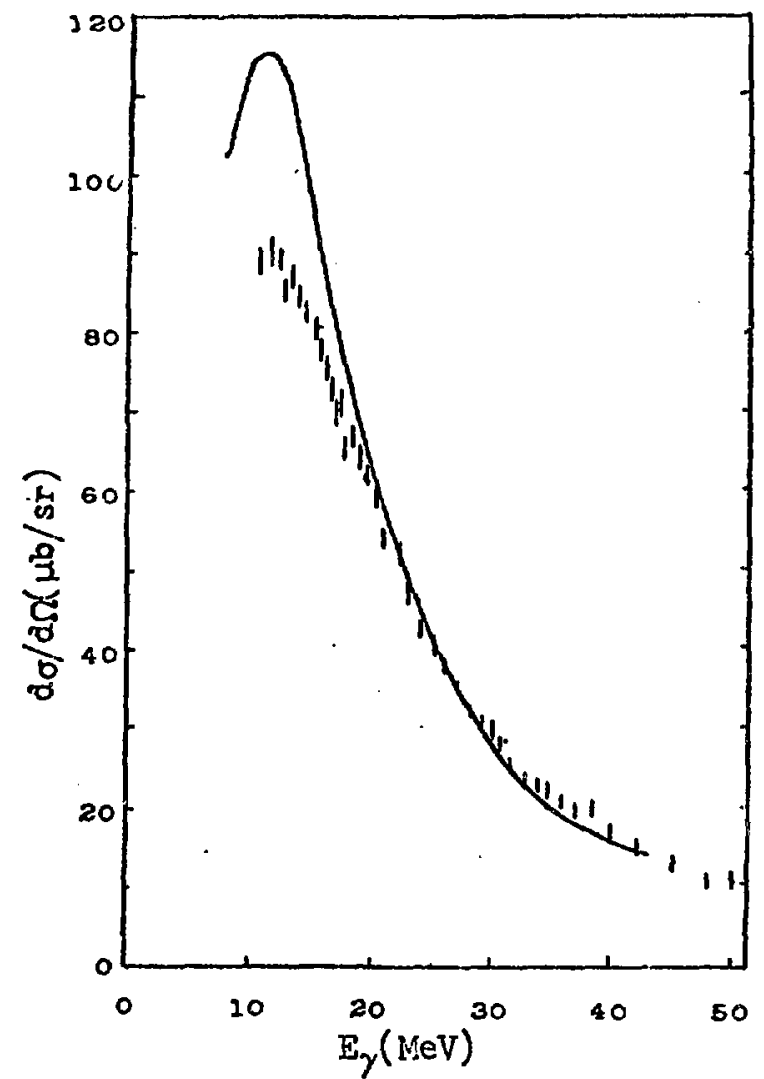

Fig. 6. Comparison of the $90^{\circ}$ differential cross section data of Ref. 33 with the serrarable-potential model calculation of Ref. 32 .

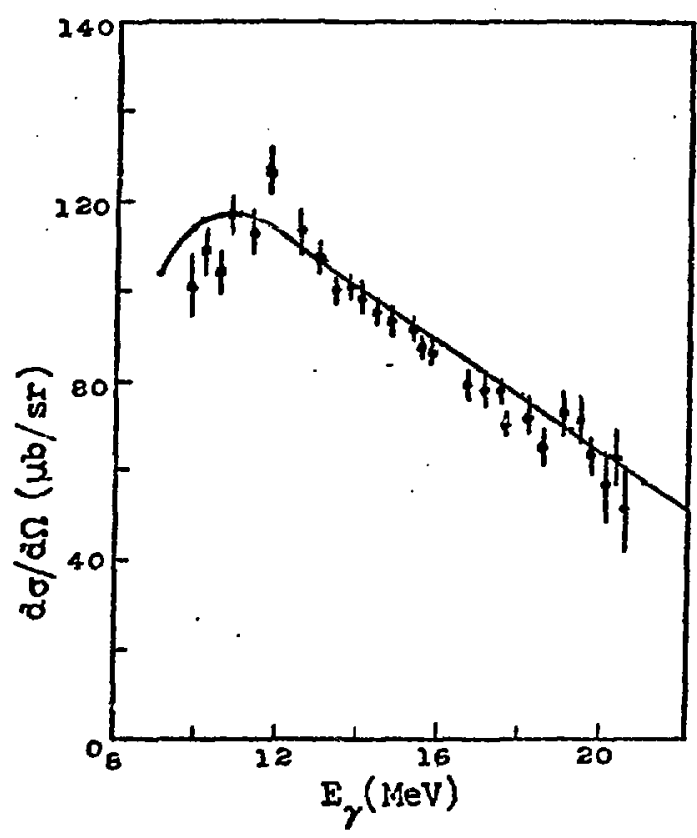

Fig. 9. Comparison of the $90^{\circ}$ differentirl cross section dat,a of Ref. 31 with the separable-potential model calculation of Ref. 32 . 
What does theory have to say? The shape of the energy spectrum is epparently well accounted for by the separable-potential model results, The cross section is governed almost entirely by the bound state energy, independent of the singlet and triplet potential assumption. This is illustrated in Fig. 10, where we plot spectra corresponding to: 1) a completeIy consistent model in which the same singlet and triplet potentials (s-wave only) are used to generate the bound state and scattering state, 2) the same model as (I) except that the bound state energy is artificially adjusted to the experimental value after the nomentum deperdence of the bound sto.te has been detarmined, 3) the same bound state as desc.ijed in (2) but with different potential parameterization in the scattering state. It is clear that models (2) and (3) give roughly the same energy spectra; they have the same binding energy in the bound state and therefore similar asymptotic behavior. From the sum rules we know that two bound state wave functions having the same rms radii will produce about the same total cross section integral. Thus, differences in the final state interactions can only shift the energy dependence of the spectra

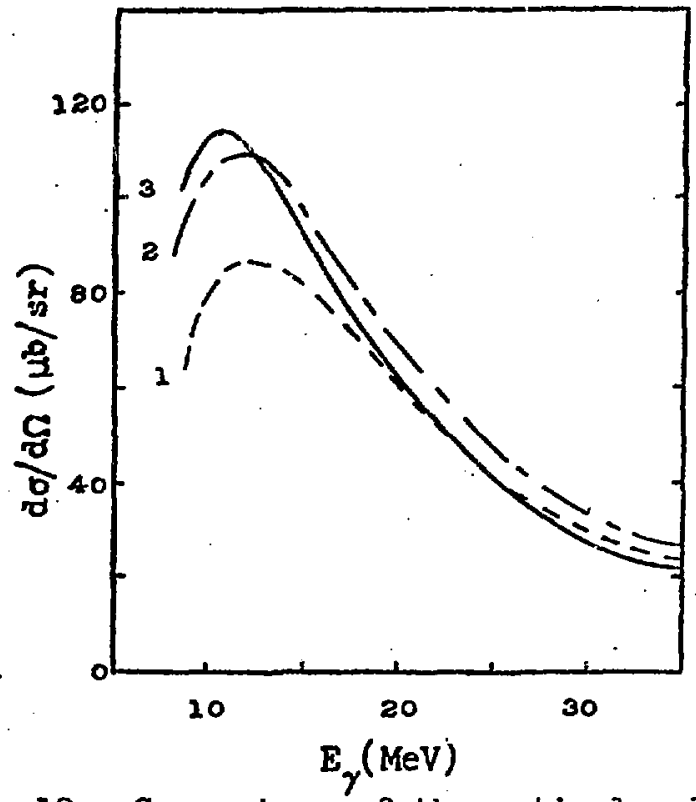

Fig. 10. Comparison of theoretical calculations of the $90^{\circ}$ differential cross section: 1) consistent model using the same potentials for the bound stote and scattering state, 2) same model as (1) but with the binding energy adjusted to the experimental value after the bound state was generated, 3) same bound state a.s (2) with different potentials used to generate the scattering stete.

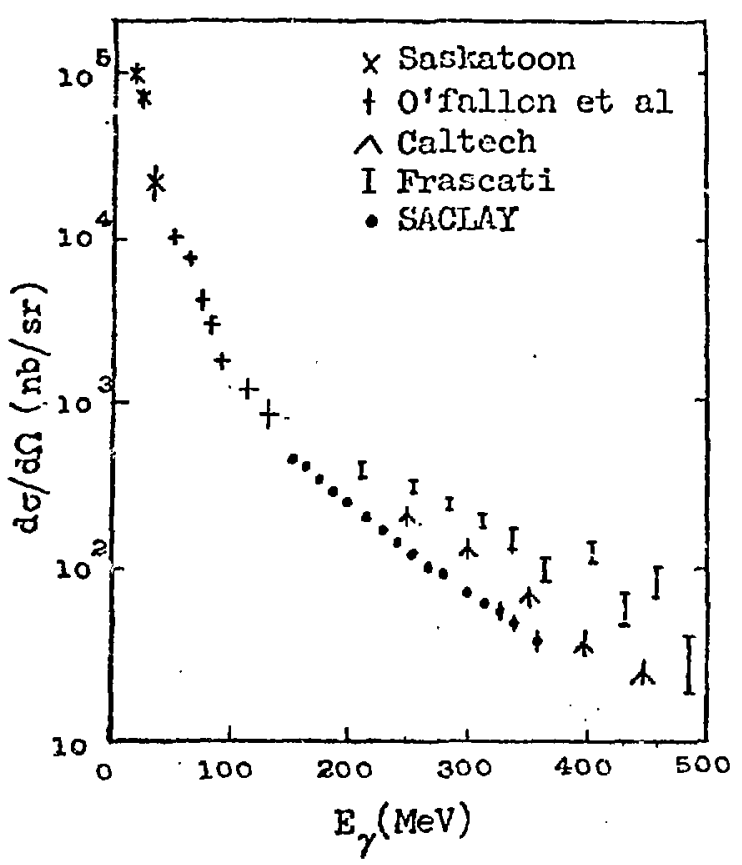

Fig. 11. Comparison of the available high energy photodinistegrulion data at $90^{\circ}$, courtesy $\mathrm{C}$. Schuhl.

slightly, as is illustrated in Fig. 10. (On the other hand, the binding energy of the bound state can change the peak cross section by a large fraction.) The contention that the separable-potential model overestimates the cross section 30 can only be checked by means of a realistic local potential calculation. However, on the basis of the available data, it vould not appear that the normalization could be off by more than $10 \%$.

The photodisintegration data for higher erergies are also interesting to examine. Besides the interesting feature in the angular distribution discussed previously, there is a change of slope in the energy spectrum above $100 \mathrm{NeV}$. In Fig. 11 is shown a comparison of the high energy date available. 35 The SACIAY data have just been obtained using a bremsstrahlung beam incident on a liquid 3 He target; both the proton and the deuteron were detected to reduce background. Note that on a log scale the data anpear to exhibit one slope below $100 \mathrm{MeV}$ and a different slope above 100 liev. Although there is some spread in the data at the higher energies, the distinct change of slope is clear. This structure of the cross section should make for interesting speculation by theorists for some time co come; it should be noted that a similar character is found for the two-body spectrum in the case of ${ }^{4} \mathrm{He}$ as opposed to the broad resonance structure 


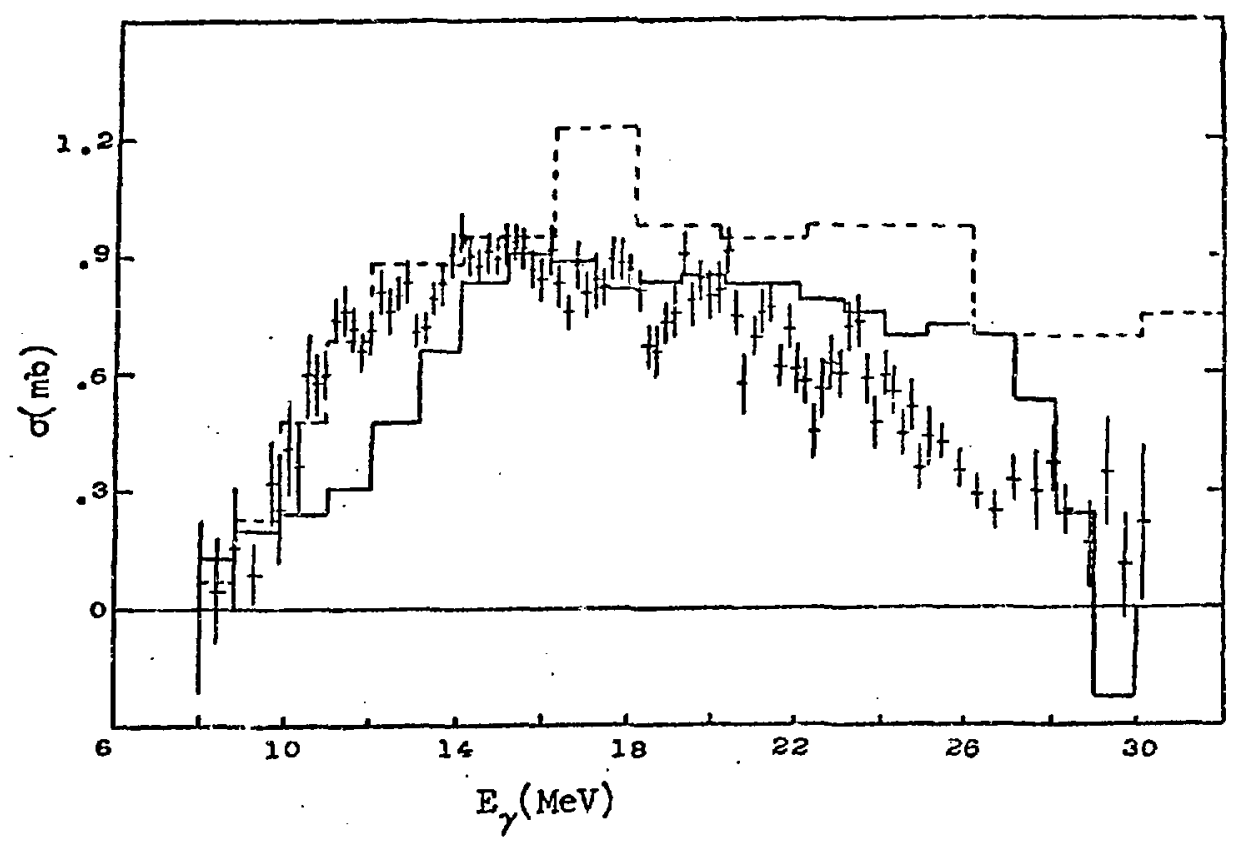

Fig. 12. Comparison of the three-body total cross section dat?: Gorbunov et al 5 ; Gerstenbers et al $\Omega$; Berman, et al $t$.

that occurs in the deuteron photodisintegration spectrum between 100 and $400 \mathrm{MeV}$.

\section{THPEE-BODY PHOTODISTITEGRATIOIT}

The photodisintegration reaction leading to the three-mucleon final state has not been extremely popular because of its difficulty. The most recont data is that of Berman et al.36 It is shown in Fig. 12, along rith the farlier measurements of Gorbunov et al ${ }^{24}$ and Gerstenberg and $O^{\prime}$ Connell.37 There anpears to be ream sonable evidence for structure of some nature in the cross section when the data of Ref. 36 are examined along with the fore-aft asymmetxy measurement of the same experiment. However, the structure would necessarily have to be relatively narrow. Theoretically any structure of such a narrow neture is hard to produce. This can be seen by looking at Lehman's formulation of the amplitude for the three-body breakup reaction diagramatically as in Fig. 13, where $X$ is the amplitude for the off-shell $i$-d scattering process and $B$ represents the Born emplitude for the reaction. 38 since $X$ is apparently smooth and the three-body finel state reaction apparentily contains little $T=\frac{1}{2}$ amplitude, it is difficult to produce much narrow structure in the theoretical cross section. In a recent review by o'Connell, 39 it was pointed uut that over the years various sharp siructure features have appeared in the trinucleon photodisintegration cross section data end that usually these features go away after a careful remeasurement and searches in alternate reactions. In light of the fate of the structure in the two-body finalstate reaction, one can only hope that the structure in the three-body final-state reaction will either be confirmed or denied by other experiments before theorists spend years trying to reproduce it in model calculations.

\section{WEAK INTERACTIONS}

liany of you are aware of the close connection between the reak ald electromagnetic intergctions. Both share the seme advantages as probes of nuclear structure. Therefore, let us look briefIy at what we have to look forward to in

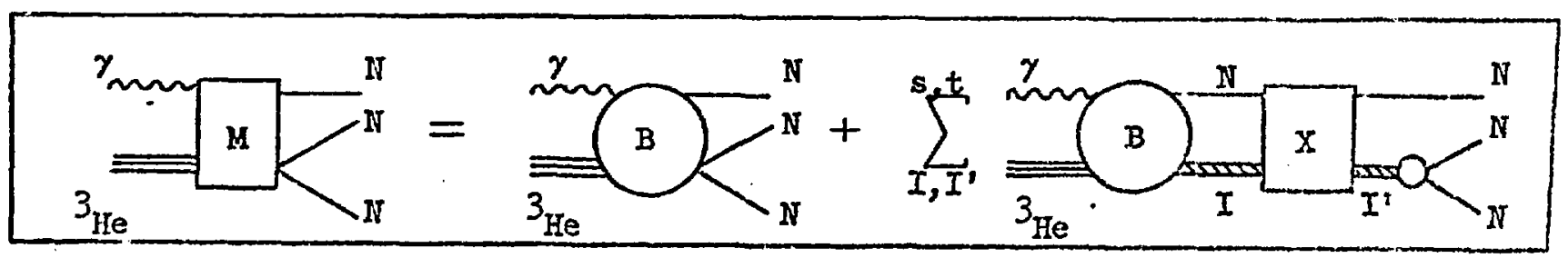

Tig. 13. A diagramatic formulation of the three-body photodisintegration amplitue? in terms of the Born emplitude for the breakup reaction and the of $\mathrm{f}-\mathrm{she} 3 \mathrm{I}$ in-d. scattering amplitude. 
the near futire. Because useful ncutrino beams, such as the one being constructed at INMP, are some distance into the future, let us concentrate on the $\mu$-capture reaction.

It should be noted that the one-body operators governing the two different processes are very similar. Instend of the familiar charce, current, and magnetic moment operators of the electromasnetic interaction

$$
\begin{aligned}
& \frac{1}{2}\left(I+\tau_{i}^{z}\right) \exp \left(i \vec{q} \cdot \vec{r}_{i}\right) \\
& \frac{1}{2}\left(1+\tau_{i}^{z}\right) \vec{p}_{i} \exp \left(i \vec{q} \cdot \vec{r}_{i}\right) \\
& \mu \vec{z}_{2}\left(1+\tau_{i}^{z}\right) \vec{\sigma}_{i} \times \vec{q} \exp \left(i \vec{q} \cdot \vec{r}_{i}\right),
\end{aligned}
$$

we have the weak vector operators wich differ from the obove in that $\left(1+\tau^{z}\right)$ is replaced by $\tau^{-}$, which transforms proton into a neutron), axial vector operators

$$
\begin{aligned}
& F_{A} \tau_{i}^{-} \vec{\sigma}_{i} \cdot \vec{p}_{i} \exp \left(i \vec{q}_{i} \cdot \vec{r}_{i}\right) \\
& F_{A} \tau_{i}^{-} \vec{\sigma}_{i} m \vec{q} \exp \left(i \vec{q} \cdot \vec{r}_{i}\right),
\end{aligned}
$$

and the psuedoscalerr operator

$$
F_{p} \tau_{i}^{\sim} \vec{\sigma}_{i} \cdot \vec{q} \exp \left(i \vec{q} \cdot \vec{r}_{i}\right)
$$

The point to be recognized is that, except for the differences in the coupling constants and the isospin changing operator $\tau^{-}$, these are the frmiliar operators whose matrix elements are measured in inelestic electron scyttering. And, as one might expect, the electric dipole transi.tion part of the $\mu$ cepture rate: (spin averaged) for

$$
\mu^{-}+A(Z, T) \rightarrow A^{*}(Z-1, T+1)+\nu_{\mu}
$$

cen be releted to tre bremsitiolung. weighted pirotonucleer sum rule for transitions to the $T+1$ giant resonance ${ }^{\text {to }}$

$$
\gamma+A(Z, T)-n^{*}(Z, T+1) \text {. }
$$

For the trinucileon sysiem there eppear to be two very interesing reactions:

$$
\begin{aligned}
& \mu^{-}+3_{\text {ILe }} \rightarrow 3_{\mathrm{H}}+\nu_{\mu} \\
& \mu^{-}+3_{\mathrm{II}} \rightarrow 3_{\mathrm{n}}+\nu_{\mu} .
\end{aligned}
$$

In the ground state to ground state reac. tion (the super-allowed transition) one can easily test coupling constants, etc. But more interesting is the possibility of using the hyperfine transition rate (3ile has spin $\frac{1}{2}$ as does the $\mu$, so that capture can occur from both $J=0$ and $\mathrm{J}=1$ states) to determine the D-state probability of the ground state rave function. The lower transition dominates and proceeds primarily through the S-state component of the wave function, whereas the upper transition is sensitive to the $\%$ D-state admixture. 4

In the three-neutron final state reaction we, of course, have the possibility of looking directly for three-neutron resonances, which re cannot do by electron scattering or photodisintegration. The neutrino will have an energy spectrum extending to approximate].y $65 \mathrm{MeV}\left[\mathrm{E}_{\max }=\right.$ $\left.2 / 3\left(m_{1}-B_{3}\right)\right]$, so that the nuclear physics in the matrix elements can be tested over a broad range of mumenta. Calculations can easily be performed in analogy with photodisintegration calculations if one uses the formulation indicated in Fig 13; only the Born amplitude need be altered.

Although the weak interactions are more alosely associated with intermediate energy physics, those working in classical photonuclear (low energy) physics should become aware of and maintain an active interest in weals interaction developments. The dichotomy between the two fielas is not great, if it exists at all.

\section{ACKMTOWLEDGMENIS}

I vish to thank D. R. Lehman and $J$. S. O'Connell for various comments relating to the trinucleon problem. Also, I am grateful to L. M. Delves, A. C. Phillips, C. Schuhl, J. I. Matthews, R. O. Owens, and H. R. Dodge for preprints and correspondence relating to their work.

* Work performed under the auspices of the U. S. Atomic Inergy Comission.

${ }^{1}$ For more detailed reviews of the general three-nucleon problem sce R. D. Amado, Ann. Rev. of Nucl. Sci. 19, 61 (1969); L. M. Delves and A. C. Philiins, Rev. llod. Phys. 41, 4.97 (1969).

${ }^{2}$ M. A. Hernell and L. M. Delves, Phys. Letters 4OB, 20 (19'72); also "The $\frac{1}{5}^{+}$State of "H and "Hic Below Breatup Threshold" preprint. 
${ }^{3} \mathrm{~J}$. Dabrowsky, E. Fedorynska, P. Hacnsel, and M. Y. M. Ifassan, Phys. Rev. Cik, 1985 (1971).

"J. A. Tjon, B. F. Gibson, and J.S. 0 'Connelz, Phys. Rev. Letters 25, 540 (1970); R. A. Malfliet and J. A. Tjon, Phys. Letters 35B, 487 (1971); Y. E. Kin and A. Tubis, Prys. Letters 38B, 354 (1972); E. Ifadjimichael, E. Harms, and V. Newton, Phys. Leiters 40B, 61 (1972); G. Gignoux and A. Laverne, Phys. Rev. Letters 29, 436 (1972); and E. Harper, Y. E. Kim, and A. Tubis, Phys. Rev. Letters 28, 1533 (1972).

${ }^{5}$ I. I. Schiff, Phys. Rev. 133, B802 (1964).

${ }^{6} \mathrm{~J}$. S. McCarthy, I. Sick, R. R. Whitney, and $M . R$. Yearian,Phys. Rev. Letters 25, 884 (1970).

7H. Collard, R. Hofstadter, F.B. Hughes, A. Johansson, M. A. Yearian, R. B. Dey, and R. T. Wagner, Phys.Rev.138, B57(1965).

${ }^{8}$. V. Reia, Ann. of Phys. 50, 4li(1968)

${ }^{9} \mathrm{~V}$. Franco, "Electron-Deuter'on Scattering and Two-Photon Exchange" preprint.

${ }^{10}$ D. R. Lehman, Phys. Rev. C3, 1827(1971.).

${ }^{11}$ S. K. Kundu, Y. M. Shin, and G.D. Wait, Nucl. Phys. Al71, 384 (1971); W. R. Dodge, J. J. Murphy, and J. M. Wyckoff, Buzl. An. Phys. Soc. 17, 152 (1972); H. R. Dodge, C. C. Chang, and J. J. Murphy, Phys. Rev. (to be published).

${ }^{1 z_{B}}$. F. Gibson and H. T. Williams, Nucl. Phys. Al63, 193 (1971) and references cited therein.

${ }^{13} \mathrm{G}$. Barto:, Ivucl. Phys. A104, 189(1967); J. S. O'Connell anü F. Prats, Phys, Rev. 184, 1007 (1969); E. Hayward, B. F. Gíbson, and J.S. O'Connell, Phys. Rev. C5, 846 (1972).

${ }^{I}$ V. N. Fetisov, A. N. Gorbunov, and A. T. Varfolomeev, Nucl. Phys. 7I, 305(1965). ${ }^{15}$ B. I. Berman, S. C. Fultz, and P. F. Yergin, Phys. Rev. Letters 2l4, 2494(1970).

${ }^{36}$ I. I. Barbour and A. C. Phillips, Phys. Rev. C1, I65 (1970).

${ }^{17}$ B. $\vec{D}$. Belt, C. R. Bingham, M. L. Halbert, and A. van der Woude, Phys. Rev. Letters 2lt, 1120 (1970).

${ }^{28} \mathrm{~B}$. F. Gibson and J.S. O'Connell, Phys. Letters 32B, 331 (1970).

${ }^{19}$ It should be noted that $\gamma / \beta^{2} \neq \frac{1}{4}$.

2ol. I. Halbert, P. Paul, K. A. Snover, and $\mathrm{E} . \mathrm{K}$. Tarburtor, International Conference on Fer Particle Problems in Iuclear Physics, UCIA (1972); S. K. Kundu, Y.M. Shin, and G. D. Wait, Hucl. Phys. A17l, 33): (1971); G. Ticcioni, S. N. Gixrdner, J. L. litthews, and R. O. Orens (contribution to this conference).

21 J. P. Didilez, H. Langevin-Joliet, $\mathrm{Z}$. Maric, ant V. Radojevic, jucl. Phys.
1143, 602 (1970); N. M. O'Palion, I. J. Koester, and T. H. Smith, Phys. Rev. C5, 1926 (1972).

${ }^{22} \mathrm{~B} . \mathrm{F}$. Gibson and J. S. O'Connell (unpublished).

${ }^{33} \mathrm{~J}$. Stewart, R. Morrison, and J. S. O'Connel1, Phys. Rev. 138, B372 (1964);

B. L. Berman, I. J. Koester, and J. H. Smith, Phys. Rev. 133, BII7 (1964).

${ }^{24} V$. N. Fetisov, $A . N$. Gorbunov, and A.I. Fetisov, Nucl. Phys. 71, 305 (1955); also references cited therein to earlier work. ${ }^{25}$ U. Eichman, Z. Physik 175, (1963); B. F. Gibson, Nucl. Fhys. B2, 501 (1967); V. N. Fetisov, Sov. J. Nucl. Phys. 4, 51 (1967).

${ }^{26}$ I. M. Barbour and A. C. Phillips, Phys. Rev. CI, 165 (1970).

27. $\vec{D}$. Belt, C. R. Bingham, M. I. Halbert, and $A$. van der Woude, Fhys, Rev. Letters 24,1120 (1970); S. K. Kundu, Y.M. Shin, and G. D. Wait, Nucl. Phys. Al7l, 384 (1971); M. I. Halbert, P. Paul, K. A. Snover, and $\mathrm{T} . \mathrm{K}$. Warburton, International Conference on Few Particle Problems in Nuclear Physics, UCIA (1972).

${ }^{28} \mathrm{~J}$. I. Matthews, T. Cruse, M. E. Orens, R. O. Owens, and W. Savin, Buzl. Am. Phys. Soc. 18, 19 (1973).

${ }^{29} \mathrm{G} . \mathrm{T}$ iccioni, S. N. Gardner, J. I.

Matthews, and $R$. 0 . Owens (contribution to this conference).

${ }^{30} \mathrm{~J} . \mathrm{A}$. Hendry and A. $\therefore$. Phillips, "The Two-Body Photodisintegration of the ${ }^{3} \mathrm{He}$. Wucleus" preprint.

${ }^{33} \mathrm{~W} . \mathrm{R}$. Dodge, C. C. Chang, and J. J. Iurphy, Phys. Rev, (to be published).

${ }^{32}$ B. F. Gibson and D. R. Lehman (unpublished).

${ }^{33} \mathrm{G}$. Ticcioni, J. I. Matthews, and R. 0 . orens, Glasgow University (private communication).

${ }^{34} \mathrm{~V}$. R. Dodge, National Bureau of Standards (private communication); see also Ref. 31.

${ }^{35}$ The data in Fig. II were graciously supplied by $C$, Schuhl, SACIAY (private communication).

${ }^{36}$ B. I. Berman, S. C. Fultz, and P. F. Yergin, Phys. Rev. Letters 24, 1494(1970).

${ }^{37} \mathrm{H}$. M. Gerstenberg and J. S. $0^{\prime}$ Connell,

Phys. Rev. 14l, 831+ (1966).

${ }^{38} \mathrm{D}$. R. Lehman, The George Washington University (private communication).

${ }^{39} \mathrm{~J}$. S. O'Connell, Conference on Electromagnetic Interactions of Nuclei at Low and Medium Inergy, Moscow (1972).

${ }^{40} \mathrm{~J}$. S. O'Connell, Buil. Am. Phys. Soc. 18 (1973).

TIJ. S. O'Connell, National Bureau of Standards (private communication). 